12. McDonagh AF, Palma LA, Trull FR, Lightner DA 1982 Phototherapy for neonatal jaundice. Configurational isomers of bilirubin. J Am Chem Soc 104:6865

13. Pratesi $R 1983$ Two lights for phototherapy. Lancet $2: 859$

14. Pratesi R, Agati G, Fusi F 1984 Configurational isomerization of bilirubin "in vitro." I. Quenching of Z-E isomerization by two-wavelength irradiation. Photochem Photobiol 40:41

15. Pratesi R, Agati G, Fusi F Configurational photoisomerization of bilirubin "In vitro." II. Comparison of laser and fluorescent lamp excitation. Photochem Photobiol (in press)

16. Pratesi R, Ronchi L, Cecchi G, Sbrana G, Migliorini MG, Vecchi C, Donzelli GP 1984 Skin optics and phototherapy of jaundice. Photochem Photobiol 40:77

17. Sbrana G, Migliorini MG, Vecchi C, Donzelli GP 1981 Laser photolysis of bilirubin. Pediatr Res 15:1517

\title{
Serum Neutral $\alpha$-D-Glucosidase from Patients with Cystic Fibrosis and Chronic Pulmonary Disease
}

\author{
JACK A. ALHADEFF, BONNIE C. POLLACK, ROBERT L. HOPFER, AND \\ DOUGLAS S. HOLSCLAW, JR. \\ Department of Chemistry, Lehigh University [J.A.A., B.C.P., R.L.H.], Bethlehem, Pennsylvania 18015 and \\ Department of Pediatrics, Hahnemann Universty [D.S.H.], Philadelphia, Pennsylvania 19102
}

\begin{abstract}
Previous studies have indicated that $\alpha$-Dglucosidase activity is increased and exhibits abnormal properties in tissues from patients with cystic fibrosis (CF). In the present investigation serum $\alpha$-D-glucosidase from patients with $\mathrm{CF}$ and from patients with chronic pulmonary disease (e.g. asthma and bronchiectasis) has been studied to determine the specificity of the $\alpha$-D-glucosidase alteration(s) in CF sera. Both groups of patients have elevated $\alpha$-D-glucosidase activity levels and similarly abnormal isoelectric focusing profiles with significantly less activity associated with acidic enzymatic forms (i.e. having isoelectric points below 4.8 ). These results suggest that the abnormalities of CF serum $\alpha$-D-glucosidase may be secondary to chronic pulmonary disease and emphasize the importance of including appropriate pathological controls in biochemical studies on CF. (Pediatr Res 19: 171-174, 1985)
\end{abstract}

\section{Abbreviations}

CF, cystic fibrosis

pI, isoelectric point

CF is one of the most common genetic diseases among Caucasian children and young adults. It manifests itself as a gener-

Received August 7, 1984; accepted September 25, 1984.

Correspondence and reprint requests should be sent to Dr. Jack A. Alhadeff, Department of Chemistry, Seeley G. Mudd Building, Lehigh University, Bethlehem, PA 18015 .

Supported in part by Cystic Fibrosis Foundation Grant G055, Cystic Fibrosis Foundation Center Grant C-099-5, and a Pediatric Pulmonary Center grant from the Bureau of Community Health Service Training Branch, Department of Health and Human Services (MCT 09020). alized disorder primarily affecting exocrine glands and the pulmonary and gastrointestinal systems (9). The major clinical findings include abnormally high sodium and chloride concentrations in sweat, pancreatic exocrine insufficiency, and chronic progressive obstructive and destructive pulmonary disease. The prognosis is poor and death usually occurs before or in early adulthood, most often as a result of respiratory failure (9). The primary biochemical defect in this lethal disease is unknown and no adequate biochemical marker for detecting heterozygous carriers is yet available.

Many biochemical studies have investigated the presence of factors, abnormal proteins and/or altered enzymes in tissues derived from patients with CF $(1,7,9)$. Numerous studies have been done on lysosomal glycosidases but few consistent findings have been reported. Among the most reproducible of the findings on glycosidases is the significant elevation of $\alpha$-D-glucosidase activity in CF tissues (2-7). In a previous study we confirmed the fact that neutral $\alpha$-D-glucosidase activity is significantly elevated in CF sera (2). In addition, we demonstrated that this increased activity was not due to 1) increased stability upon storage at $-20^{\circ} \mathrm{C}, 2$ ) the presence of activators in CF sera or inhibitors in control sera, or 3) kinetic differences of the $\alpha$-Dglucosidases. The major apparent difference between $C F$ and control sera $\alpha$-D-glucosidases was revealed in isoelectric focusing studies which indicated that significantly less $\mathrm{CF} \alpha$-D-glucosidase activity was associated with enzymatic forms with pI's below 4.8.

In the present investigation additional studies have been performed on serum $\alpha$-D-glucosidase from patients with CF and from patients with chronic pulmonary disease (without $C F$ ) to determine the specificity of the $\alpha$-D-glucosidase alteration(s). The results indicate that both groups of patients have elevated serum $\alpha$-D-glucosidase activity levels and similarly abnormal isoelectric focusing profiles, suggesting that the abnormalities may be secondary to chronic pulmonary disease. 


\section{MATERIALS AND METHODS}

General. All procedures were carried out at $0-4^{\circ} \mathrm{C}$ unless otherwise stated. 4-Methylumbelliferyl- $\alpha$-D-glucopyranoside was purchased from Sigma Chemical Co. and used for assaying $\alpha-\mathrm{D}$ glucosidase activity.

Tissue preparation. Whole blood was drawn from normal controls and from patients with CF and a variety of chronic lung diseases (asthma, bronchiectasis, immotile cilia syndrome, etc.) followed at the Pediatric Pulmonary and Cystic Fibrosis Centers, Hahnemann University, Philadelphia, PA (see Table 1). Sera were prepared from whole blood as follows: the whole blood was allowed to coagulate at room temperature for 30-60 min, centrifuged at $2000 \times g$ for $20 \mathrm{~min}$, and the resultant soluble sera were poured into separate glass tubes and stored frozen $\left(-20^{\circ} \mathrm{C}\right)$ until used. The procedures which involved human' subjects were approved by an institutional Committee on Investigations/Activities Involving Human Subjects. Informed consent was obtained from all subjects in this study.

$\alpha$-D-Glucosidase assay. Neutral $\alpha$-D-glucosidase activity in sera and in the isoelectric focusing fractions was determined at pH 5.9 using $0.1 \mathrm{M}$ citric acid-0.2 $\mathrm{M} \mathrm{Na}_{2} \mathrm{HPO}_{4}$ buffer. Aliquots $(10-20 \mu \mathrm{l})$ of undiluted sera were brought to a volume of $50 \mu \mathrm{l}$ with the citrate-phosphate buffer and the reactions were initiated with $50 \mu$ l of $2 \mathrm{mM} 4$-methylumbelliferyl- $\alpha$-D-glucopyranoside

Table 1. Patient information and serum $\alpha-D-$-Glucosidase activities

\begin{tabular}{lcccc}
\hline & & & & $\alpha$-D-Glucosidase \\
activity \\
Patients & $\begin{array}{c}\text { Age } \\
\text { (yr) }\end{array}$ & Sex & $\begin{array}{c}\text { Clinical } \\
\text { score (8) }\end{array}$ & $\begin{array}{c}\text { (units/ml sera) } \\
\text { CF }\end{array}$ \\
EP* & 29 & M & 60 & 22.0 \\
EG $\dagger$ & 40 & M & 47 & 8.8 \\
LW & 32 & F & 78 & 7.7 \\
TL & 22 & M & 34 & 19.5 \\
AG & 19 & M & 66 & 19.2 \\
CM & 33 & M & 58 & 14.8 \\
WC* & 22 & M & 59 & 13.9 \\
JC† & 27 & M & 72 & 22.2 \\
LV* & 21 & F & 48 & 38.2 \\
GC $\dagger$ & 38 & M & 66 & 12.7 \\
GD & 28 & M & 70 & 13.7 \\
DG & 23 & F & 90 & 4.5 \\
JD & 25 & M & 75 & 5.6 \\
SN & 30 & M & & 19.6 \\
& & & & $15.8 \pm 8.7 \S$
\end{tabular}

Chronic pulmonary diseases (diagnosis)

$\begin{array}{lllr}\text { BE (asthma) } & 12 & \mathrm{~F} & 4.7 \\ \text { FR (asthma) } & 21 & \mathrm{M} & 12.9 \\ \text { KM (asthma) } & 19 & \mathrm{~F} & 9.1 \\ \text { RG (asthma) } & 14 & \mathrm{M} & 5.8 \\ \text { JN (asthma) } & 20 & \mathrm{M} & 12.8 \\ \text { DM (asthma) } & 16 & \mathrm{M} & 5.9 \\ \text { JT (asthma) } & 17 & \mathrm{M} & 2.5 \\ \text { RW (bronchiectasis) } & 18 & \mathrm{M} & 6.8 \\ \text { EB (bronchiectasis) } & 18 & \mathrm{~F} & 11.6 \\ \text { CY (bronchiectasis) } & 12 & \mathrm{~F} & 6.9 \\ \text { RT (bronchiectasis) } & 17 & \mathrm{~F} & 9.3 \\ \text { RB (chronic lung disease) } & 15 & \mathrm{M} & 25.1 \\ \text { AV (pigeon handler's dis- } & 15 & \mathrm{M} & 6.4 \\ \quad \text { ease) } & & & \\ \end{array}$

* Patients without pancreatic insufficiency. $\S$ Patients who also have diabetes mellitus. $\ddagger$ Patients who also have biliary cirrhosis. $\S$ Mean \pm SD.

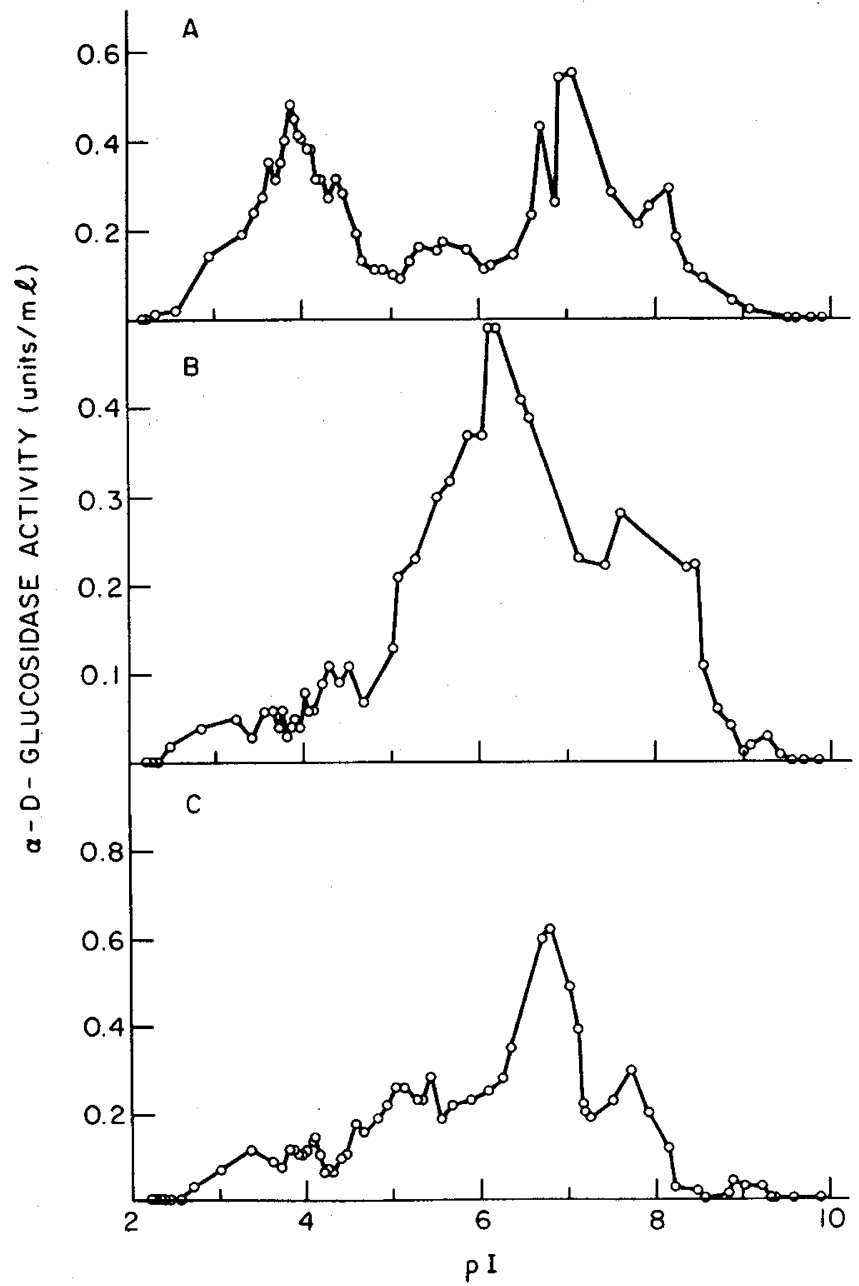

Fig. 1. Isoelectric focusing profiles of serum neutral $\alpha$-D-glucosidase activity from: $A$, normal control (female, $21 \mathrm{yr}$ old); $B$, patient with $C F$ (AG) with pancreatic insufficiency; $C$, patient with CF (EP) without pancreatic insufficiency. See "Materials and Methods" section for details.

(in citrate-phosphate buffer). The reactions were run for 75-180 min, terminated with $1.0 \mathrm{ml} 0.085 \mathrm{M}$ glycine-carbonate buffer (pH 9.7), and fluorescence read on a Turner fluorometer. These activity readings were corrected for tissue and substrate blanks. The assay was linear for amount of protein and for incubation time under the above conditions. A unit of $\alpha$-D-glucosidase activity was defined as the amount of enzyme necessary to hydrolyze $1 \mathrm{nmol}$ of substrate $/ \mathrm{h}$ at $37^{\circ} \mathrm{C}$.

Isoelectric focusing. Isoelectric focusing was performed essentially as previously described (2) using $2 \%$ (v/v) pH 4-6 ampholytes (LKB Produkter) in a gradient of $0-67 \%(\mathrm{w} / \mathrm{v})$ sucrose on a $40.0 \mathrm{ml}$ analytical column. Control and patient sera $(0.3-$ $3.0 \mathrm{ml}$ ) were subjected to isoelectric focusing at a starting voltage of $600 \mathrm{~V}$ and current of 2.5-7.0 mA. After focusing for 13-20 h (until constant current is achieved) the column was eluted, fractions (approximately $0.3 \mathrm{ml}$ ) were collected, their $\mathrm{pH}$ values determined on a Beckman digital $\mathrm{pH}$ meter, and $50 \mu \mathrm{l}$ aliquots were assayed for neutral $\alpha-\mathrm{D}$-glucosidase activity. The isoelectric focusing profiles are plotted as amount of $\alpha$-D-glucosidase activity (units $/ \mathrm{ml}$ ) versus $\mathrm{pI}$ of the various forms of the enzyme.

Categorization of patients. The diagnosis of CF was established on the basis of characteristic clinical features and an elevated sweat chloride concentration obtained by the quantitative pilocarpine iontophoresis method. All patients were clinically stable at the time blood samples were drawn. Their ages ranged from $19-40 \mathrm{yr}$ and the clinical severity of their illness, using a standardized system of scoring $(8)$, ranged from $34-90(0=$ worst; 100 


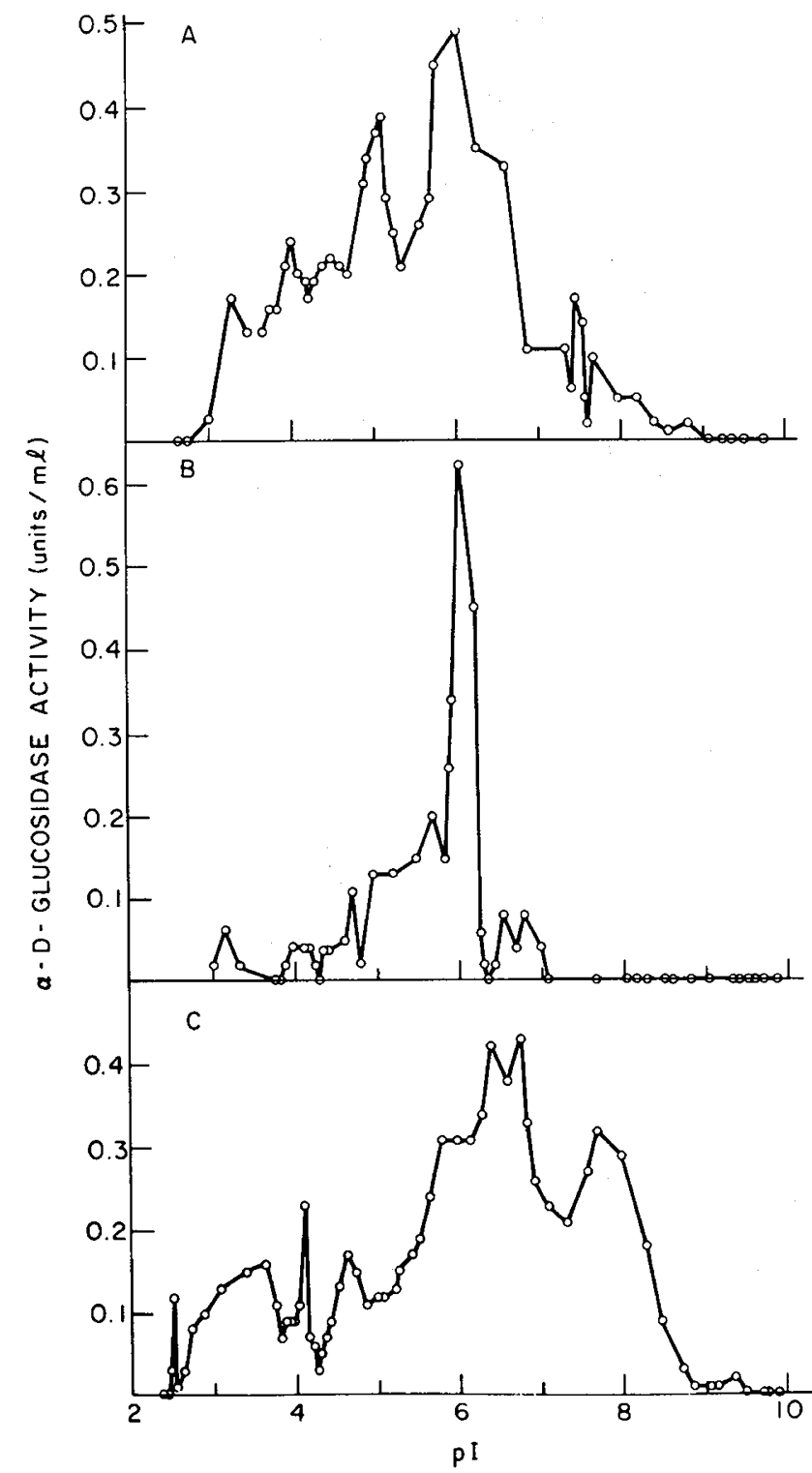

Fig. 2. Isoelectric focusing profiles of serum neutral $\alpha$-D-glucosidase activity from chronic pulmonary disease patients: $A$, asthma (patient $\mathrm{DM}$ ); $B$, bronchiectasis (patient $\mathrm{RT}$ ); $C$, chronic lung disease (patient RB). See "Materials and Methods" section for details.

$=$ best). Patients were chosen to include those with and without pancreatic insufficiency and with and without diabetes mellitus. One patient had biliary cirrhosis and one had multiple chronic inflammatory giant pancreatic cysts. All were receiving oral antibiotics and those with pancreatic insufficiency were taking pancreatic enzyme supplements.

Statistical tests. The nonparametric Mann-Whitney $U$ test was employed to determine if the mean serum $\alpha$-D-glucosidase activity levels were significantly different in the three groups $(C F$, chronic lung disease, and normal control).

\section{RESULTS}

Table 1 summarizes the host information (e.g. diagnosis, sex, age), clinical scores, and serum $\alpha$-D-glucosidase activities of the patients investigated in the present study. The mean serum $\alpha-D$ glucosidase activity was somewhat higher in the patients with $\mathrm{CF}$ $(15.8 \pm 8.7$ units $/ \mathrm{ml})$ than in the chronic lung disease patients $(9.2 \pm 5.7$ units/ml) and a wide range of activity was found for both groups. These mean activities are both significantly higher $(p<0.002)$ than the mean $\alpha$-D-glucosidase activity $(2.7 \pm 0.8$ units/ml) of 15 normal controls found in our previous study (2).

Figure $1 A$ depicts a representative isoelectric focusing profile of serum $\alpha$-D-glucosidase from a normal control. Two major areas of approximately equal enzymatic activity are observed centered around pI values of 4.0 and 7.0. As in our previous study (2), a large percentage of the total recovered $\alpha$-D-glucosidase activity in control sera exists below a pI value of 4.8 . In contrast, Figure $1 B$ and $C$ indicate that patients with $C F$ both with (patient AG) and without (patient EP) pancreatic insufficiency, respectively, have serum $\alpha$-D-glucosidase profiles with little activity below $\mathrm{pI}$ values of 4.8 . These profiles are representative of the great majority of patients with $\mathrm{CF}$ investigated in the present and previous study (2). Three of the patients with CF (EG, GC, and JD, Table 1) had serum $\alpha$-D-glucosidase profiles very similar to normal controls (as in Fig. $1 A$ ) with large relative amounts $(\geq 50 \%)$ of total recovered activity below a pI value of 4.8. Two of these patients (EG and GC) also have diabetes mellitus but this is unlikely to account for a normal-appearing $\alpha$-D-glucosidase profile since a third patient with $\mathrm{CF}$ (JC) with diabetes mellitus gave the characteristic atypical profile.

Figure 2 depicts serum $\alpha$-D-glucosidase profiles of three pathological controls, i.e. patients without $\mathrm{CF}$ but with chronic pulmonary disease. The profile in Figure $2 A$ of a patient (DM) with asthma is representative of seven asthma patients investigated and the profile in Figure $2 B$ (patient RT) is representative of the four bronchiectasis patients investigated. The profile from a patient (RB) with chronic bronchocentric granulomatous lung disease is given in Figure $2 C$. Inspection of all the profiles in Figure 2 indicates that the great majority of enzymatic activity in these pathological controls occurs at $\mathrm{pI}$ values greater than 4.8 (as it does in the CF profiles). Atypical $\alpha$-D-glucosidase profiles (similar to those in Fig. 2) were found for 12 of 13 of the patients with chronic pulmonary disease.

\section{DISCUSSION}

Several previous studies have documented abnormalities in the activity levels and/or properties of $\alpha$-D-glucosidase derived from CF tissues (2-7). In one study isoelectric focusing revealed consistently decreased relative amounts of $\alpha$-D-glucosidase activity with acidic pI values $(\leq 4.8)$ in CF sera when compared to control sera (2). In the present investigation this earlier study has been extended to include 14 additional patients with $\mathrm{CF}$ as well as 13 pathological controls suffering from chronic pulmonary disease. The chronic pulmonary disease patients without $\mathrm{CF}$ were included to determine the specificity of the $\alpha$-D-glucosidase alterations in CF sera since essentially all patients with CF suffer from chronic lung disease (9). As in the previous study, increased $\alpha$-D-glucosidase activity levels and altered isoelectric focusing profiles with decreased relative amounts of acidic forms have been found in CF sera. These $\alpha$-D-glucosidase alterations do not appear to be related to any of a number of CF patient host factors (e.g. sex, age, severity of disease, presence of pancreatic insufficiency, or diabetes mellitus) but the data are too limited for any definite conclusions in this regard.

The chronic pulmonary disease patients without CF had an increased mean serum activity level of $\alpha$-D-glucosidase but not as high as in the patients with CF. These patients also exhibited altered $\alpha$-D-glucosidase profiles (with decreased amounts of activity with acidic pI values) similar to those seen in sera from patients with CF. As a matter of fact, the profiles in the patients with chronic pulmonary disease were more consistently altered than in the patients with CF. Only one (RW) of 13 of these patients had a serum $\alpha$-D-glucosidase profile with a large amount ( $\geq 50 \%$ ) of activity with pI values below 4.8 . Interpretation of this profile is complicated by the fact that this patient has immune deficiency in addition to bronchiectasis. The findings in the pathological controls suggest that the alterations of serum $\alpha$-D-glucosidase in patients with CF may be secondary to chronic 
pulmonary disease. The alterations are unlikely to be secondary to the pancreatic insufficiency found in the great majority of patients with CF since the three patients without pancreatic insufficiency also exhibited increased $\alpha$-D-glucosidase activity levels and altered isoelectric profiles.

The results of the present investigation emphasize the importance of including appropriate pathological controls in addition to normal controls when doing biochemical studies on CF. Since appropriate pathological controls are often not included in studies on CF, it is likely that many of the biochemical abnormalities found in tissues derived from patients with CF are not specific for $\mathrm{CF}$ but are secondary to chronic disease.

Acknowledgment. Deborah Christie, R.N., organized and participated in the collection of patient blood samples.

\section{REFERENCES}

1. Alhadeff JA 1980 Glycoprotein metabolism in cystic fibrosis. In: Sturgess JM (ed) Perspectives in Cystic Fibrosis. Proceedings of the Eighth International Congress on Cystic Fibrosis. Imperial Press, Ontario, pp 35-43
2. Alhadeff JA, Thom D, Holzinger RT 1981 Activity levels and properties of acid $\alpha$-glucosidase from liver and neutral $\alpha$-glucosidase from sera of cystic fibrosis patients and controls. Clin Chim Acta 117:227-237

3. Antonowicz I, Sippell WG, Shwachman H 1972 Cystic fibrosis: lysosomal and mitochondial enzyme activities of lymphoid cell lines. Pediatr Res 6:803812

4. Casola L, DiMatteo G, Romano M, Rutigliano B, Mastella G 1979 Glycosidases in serum of cystic fibrosis patients. Clin Chim Acta 94:83-88

5. DiMatteo G, Romano M, Mastella G, Castellani E, Rutigliano B, Casola L 1979 Chemical comparison of normal and cystic fibrosis meconium: quantitative and qualitative analysis of carbohydrate splitting enzymes. Monogr Pediatr 10:19-26

6. Hosli P, Vogt E 1977 Cystic fibrosis: leakage of lysosomal enzymes and of alkaline phosphatase into the extracellular space. Biochem Biophys Res Commun 79:741-748

7. Riordan JR, Alon N, Duthie M, Maler T, Buchwald M 1980 Hydrolytic enzymes as markers for cystic fibrosis. In: Sturgess JM (ed) Perspectives in Cystic Fibrosis. Proceedings of the Eighth International Congress on Cystic Fibrosis. Imperial Press, Ontario, pp 313-321

8. Shwachman H, Kulczycki LL 1958 Long-term study of one hundred five patients with cystic fibrosis. Am J Dis Child 96:6-15

9. Talamo RC, Rosenstein BJ, Berninger RW 1983 Cystic fibrosis. In Stanbury JB, Wyngaarden JB, Frederickson DS, Goldstein JL, Brown MS (eds) The Metabolic Basis of Inherited Disease, 5th ed. McGraw-Hill, New York, pp 1889-1917

\title{
Day-to-Day Pneumogram Variability
}

\author{
CARL E. HUNT, ROBERT T. BROUILLETTE, KIANG LIU, AND LINDA KLEMKA \\ Departments of Pediatrics, Community Health and Preventative Medicine, Northwestern University Medical \\ School and Children's Memorial Hospital, Chicago, Illinois 60614
}

\begin{abstract}
To determine day-to-day variability in respiratory pattern, we obtained 188 comparisons of pneumograms performed during two successive 24-h intervals. The respiratory pattern values calculated were total duration of brief apnea (apnea density), periodic breathing episodes, longest apnea, number of apneas >11 s, and number of apneas $>15 \mathrm{~s}$. For day 1 values for apnea density, periodic breathing, and longest apnea which were within the 90th percentile for normal infants at age 1 month, day 2 values fell outside that range in $4.3,3.5$, and $18 \%$ of comparisons, respectively. There was a systematic tendency for the day 2 values to be less than the day 1 values. For each parameter, the day 2 range was lower, the slope of the day 2 versus day 1 regression line was significantly less than $1(p<0.01)$ and the $y$-intercept was significantly greater than zero $(p<0.01)$. Knowledge of day-to-day pneumogram variability should be helpful in interpreting individual pneumogram results and in assessing the clinical usefulness of pneumogram recordings. (Pediatr Res 19: 174-177, 1985)
\end{abstract}

Received November 7, 1983; accepted September 27, 1984.

Address for reprints Carl E Hunt, M.D., Division of Neonatology, Children's Memorial Hospital, 2300 Children's Plaza, Chicago, IL 60614.

Supported by the Children's Research Guild and the Lillian S. Wells Foundation.

\begin{abstract}
Abbreviations
SIDS, sudden infant death syndrome

AOI, apnea of infancy

$\mathbf{A}_{6} / \mathbf{D} \%$, apnea density
\end{abstract}

Two-channel recordings of ECG and thoracic impedence (pneumograms) are commonly performed in infants with symptomatic apnea and in asymptomatic infants considered to be at increased risk for SIDS. Based primarily on single 24-h recordings, Kelly and coworkers $(3,4)$ documented significantly greater periodic breathing in AOI infants and in subsequent siblings compared to control infants. In earlier reports, we confirmed the presence of pneumogram abnormalities in AOI compared to control infants (2), and documented improvement in these abnormalities with theophylline (1).

Investigators have used various methods for printing and displaying pneumogram recordings and different criteria for pneumogram interpretation. In addition to uncertainty as to the upper normal limit for the various calculated parameters, there are no reported studies of day-to-day variability in pneumogram results. Although two previous pneumogram studies in normal infants $(2,5)$ did include some serial recordings, variability could 